\title{
THEOREMS ON NULL-PATHS AND REDSHIFT
}

\author{
M. I. WANAS ${ }^{1,3}$ AND A. B. Morcos ${ }^{2,3}$ \\ 1 Astronomy Department, Faculty of Science, Cairo University, Giza, Egypt \\ E-mail : wanas@frcu.eun.eg \\ 2 Astronomy Department, National Research Institute of Astronomy and Geophysics, Helwan Cairo, Egypt \\ E-mail : fadymorcos@hotmail.com,fadymorcos@nriag.sci.eg \\ ${ }^{3}$ Center of Theoretical Physics(CTP), British University(BUE), Egyptian Relativity Group, Egypt \\ (Received February 17, 2013; Revised May 1, 2013; Accepted May 1, 2013)
}

\begin{abstract}
In the present work, we prove the validity of two theorems on null-paths in a version of absolute parallelism geometry. A version of these theorems has been originally established and proved by Kermak, McCrea and Whittaker (KMW) in the context of Riemannian geometry. The importance of such theorems lies in their applications to derive a general formula for the redshift of spectral lines coming from distant objects. The formula derived in the present work can be applied to both cosmological and astrophysical redshifts. It takes into account the shifts resulting from gravitation, different motions of the source of photons, spin of the moving particle (photons) and the direction of the line of sight. It is shown that this formula cannot be derived in the context of Riemannian geometry, but it can be reduced to a formula given by KMW under certain conditions.
\end{abstract}

Key words : Null-Geodesics — Riemannian Geometry — Absolute Parallelism Geometry — Null-Paths - Redshift

\section{INTRODUCTION}

In the context of the general theory of relativity (GR), the redshift in the spectra of distant objects is a metric phenomena. In other words, knowing the metric of spacetime, one can calculate the redshift whether it is astrophysical or cosmological. An alternative and more general method for calculating the redshift is to use the results of theorems on null geodesics, established in Riemannian geometry, by Kermack, McCrea, and Whittaker (1933, hereafter KMW). It is worth mentioning that the applications of the two methods, in the context of GR, give identical results. In both methods, it is assumed that the trajectory of a photon in a gravitational field is a null geodesic. This implies the neglect of the effect of spin of the photon on its trajectory. In calculating the redshift, the first method is easier and more direct than the second, and since the two methods are equivalent in the context of GR, authors usually use the first method neglecting the second one.

Recently, some pieces of evidence indicating probable dependence of trajectories of spinning particles on their spin were reported. The first one is on the laboratory scale, which is the discrepancy between theoretical calculations and the results of the COW-experiment (Overhauser \& Colella 1974; Colella et al. 1975) and Werner et al. (1988). The second one is on the galactic scale concerning the arrival times of photons, neu- trinos (and gravitons!) from the supernova SN1987A (Schramm \& Truran 1990; Weber 1994; De Rujula 1987). As it is well known that, the idea of Parameterized Absolute Parallelism (PAP) is to hypothesize a set on n-vectors in an n-dimensional space time, each of which has a vanishing covariant derivative (the absolute parallelism condition). These vectors are then used as n- bins for an n-dimensional metric and in turn from the foundation of an extended geometry with simultaneously non-vanishing curvature and torsion. Wanas (1998) suggested a new path equation in the parameterized absolute parallelism (PAP) geometry to compute the trajectory of a spinning particle in a gravitational field. Use of these equations which depend on the spin of the particle has provided a satisfactory interpretation of the discrepancy in the COW experiment Wanas et al. (2000), and can account for the time delay of spinning particles coming from SN1987A (Wanas et al. 2002). If we use this equation to describe the trajectory of photons that are spin one massless particles, we encounter a problem concerning redshift calculations. That is, the metric of space-time is not the first integral of the new path equation and the redshift is no longer a metric phenomena. Consequently, one can not calculate the redshift using any of the above mentioned methods. One way to solve this problem is to develop theorems on the null path, similar to those of KMW theorems, in order to apply them for obtaining the redshift extracted from spinning particles. This is the aim of the present work. 
For this aim, we give a brief account on KMW theorems in Section 2. In Section 3, we review the main features of the spin dependent path equation. The validity of the KMW theorems is proved in PAP geometry in Section 4. In Section 5, we derive a general formula for redshift, taking into account the spin of the particle from which we extract the redshift. The work is discussed in Section 6.

\section{KMW THEOREMS}

The following theorems on null geodesics were established in KMW in Riemannian space $R_{n}$ of dimensions $n$, whose metric is given by,

$$
d S^{2}=g_{\mu \nu} d x^{\mu} d x^{\nu} .
$$

Consider the null geodesic $\Gamma$ connecting the two neighboring $C_{0}$ and $C_{1}$ in $R_{n}$. The tangent null vector (transport vector in KMW) is defined by

$$
\eta^{\rho} \stackrel{\text { def }}{=} \frac{d x^{\rho}}{d \lambda}
$$

where $\lambda$ is a parameter characterizing $\Gamma$. The equation for $\Gamma$ is given by

$$
\frac{d \eta^{\rho}}{d \lambda}+\left\{\begin{array}{c}
\rho \\
\mu \nu
\end{array}\right\} \eta^{\mu} \eta^{\nu}=0
$$

where $\left\{\begin{array}{c}\rho \\ \mu \nu\end{array}\right\}$ is the Christoffel symbol of the second type. Eq. 3 follows from the Euler-Lagrange equation,

$$
\frac{d}{d \lambda}\left(\frac{\partial T}{\partial \eta^{\mu}}\right)-\frac{\partial T}{\partial x^{\mu}}=0,
$$

upon taking,

$$
T \stackrel{\text { def }}{=} \frac{1}{2} g_{\mu \nu} \eta^{\mu} \eta^{\nu}
$$

Let $\Gamma^{\prime}$ be a null geodesic parallel to $\Gamma$ and passing through the point $C^{\prime}$ near $C$, and let $\xi^{\sigma}$ denote the vector $C C^{\prime}$. Let a scalar $J$ be defined as

$$
J \stackrel{\text { def }}{=} \eta_{\alpha} \xi^{\alpha}
$$

where $\eta_{\alpha}$ is the covariant form of the vector $\eta^{\alpha}$.

The KMW -Theorems can be stated as follows,

\section{Theorem (I)}

"The scalar J given by Eq. 6 is independent of the choice of the direction $C C^{\prime}$, and is also independent of the position of $C$ on the null geodesic $\Gamma$."

It depends only on the two null geodesics as a whole and not on any particular point on them. This is the first theorem which was rigorously proved by KMW.
Consider a null geodesic parallel to $\Gamma$, of which there are $\infty^{n-1}$. If we form the scalar " $J$ " corresponding to $\eta^{\mu}$ and any of these null geodesics, we find that for any particular value of " $J$ " there are $\infty^{n-2}$ parallel null geodesics.

\section{Theorem (II)}

"If the set of $\infty^{n-2}$ null geodesics lies in a $R_{n-1}$ which intersects a local flat subspace $E_{n-1}$, at $C$, in a local flat subspace $E_{n-2}$, then $E_{n-2}$ is perpendicular to the projection of $\Gamma$ in $E_{n-1}$."

As a consequence of these two theorems KMW were able to derive the following formula for the redshift extracted from photons, assuming that a photon is moving along null geodesic of the metric between the emitter at $C_{1}$ and receiver at $C_{0}$,

$$
Z=\frac{\Delta \lambda}{\lambda}=\frac{\left[\rho_{\mu} \eta^{\mu}\right]_{C_{1}}-\left[\varpi_{\mu} \eta^{\mu}\right]_{C_{0}}}{\left[\varpi_{\mu} \eta^{\mu}\right]_{C_{0}}}
$$

where $\rho_{\mu}, \varpi_{\mu}$ are the covariant form of the tangents to the geodesics of the observers at $C_{1}, C_{0}$, respectively.

\section{SPIN-DEPENDENT PATH EQUATION}

It is well known that Riemannian geometry possesses two types of the paths. The first is the geodesic path and the second is the null geodesic. The equation of these two paths can be written in the general form,

$$
\frac{d^{2} x^{\mu}}{d p^{2}}+\left\{\begin{array}{c}
\mu \\
\alpha \beta
\end{array}\right\} \frac{d x^{\alpha}}{d p} \frac{d x^{\beta}}{d p}=0,
$$

where $p$ is a parameter characterizing the trajectory of a massive or massless particle (cf., Adler et al. 1975). This parameter may be related to the parameter $S$ in Eq. 1 by

$$
d S^{2}=E d p^{2}
$$

where $E$ is a numerical parameter taking the values

$$
\begin{aligned}
& E=0, \text { for a null geodesic } \\
& E=1, \text { for a geodesic } .
\end{aligned}
$$

Wanas et al. (1995) directed their attention to the absolute parallelism space (AP-space), and by generalizing the method given by Bazanski (1977, 1989), they derived the following set of three path equations:

$$
\begin{gathered}
\frac{d J^{\mu}}{d S^{-}}+\left\{\begin{array}{c}
\mu \\
\nu \sigma
\end{array}\right\} J^{\nu} J^{\sigma}=0 \\
\frac{d W^{\mu}}{d S^{o}}+\left\{\begin{array}{c}
\mu \\
\nu \sigma
\end{array}\right\} W^{\nu} W^{\sigma}=-\frac{1}{2} \Lambda_{(\nu \sigma)} \cdot{ }^{\mu} W^{\nu} W^{\sigma}, \\
\frac{d V^{\mu}}{d S^{+}}+\left\{\begin{array}{c}
\mu \\
\nu \sigma
\end{array}\right\} V^{\nu} V^{\sigma}=-\Lambda_{(\nu \sigma) \cdot} \cdot{ }^{\mu} V^{\nu} V^{\sigma},
\end{gathered}
$$

where $J^{\mu}, W^{\mu}$ and $V^{\mu}$ are the tangent vectore to the corresponding phathes whose parameters are $S^{-}, S^{0}$ 
and $S^{+}$, respectively. Also $\Lambda_{\mu \nu}^{\alpha}$ is the torsion of the AP geometry defined by

$$
\Lambda_{\mu \nu}^{\alpha} \stackrel{\text { def }}{=} \Gamma_{. \mu \nu}^{\alpha}-\Gamma_{. \nu \mu}^{\alpha}
$$

where $\Gamma_{. \nu \mu}^{\alpha}$ is the non-symmetric affine connection defined as a consequence of the condition for AP.

Wanas (1998) defined a general expression for a connection formed by taking linear combinations of the available connections in the AP space. He mentioned that the metricity condition is necessary but not sufficient to define the Christoffel symbol. He generalized the three path Eqs. 11, 12, and 13 in the following equation,

$$
\frac{d Z^{\mu}}{d \tau}+\left\{\begin{array}{c}
\mu \\
\nu \sigma
\end{array}\right\} Z^{\nu} Z^{\sigma}=-b \Lambda_{(\nu \sigma)} \cdot{ }^{\mu} Z^{\nu} Z^{\sigma}
$$

Here, $b$ is a parameter given by $b=\frac{n}{2} \alpha \beta$ with $n(=$ $0,1,2, \ldots,), \alpha$ and $\beta$ being a natural number, the fine structure constant and a numerical free parameter, respectively. We also have $Z^{\mu} \stackrel{\text { def }}{=} \frac{d X^{\mu}}{d \tau}$, with $\tau$ being the evolution parameter along the new general path (15) associated with the general connection:

$$
\nabla_{. \mu \nu}^{\alpha}=\left\{\begin{array}{c}
\alpha \\
\mu \nu
\end{array}\right\}+b \gamma_{. \mu \nu}^{\alpha}
$$

The geometric structure characterized by the connection in Eq. 16 is called the PAP geometry (Wanas 2000). It is worthy of mention that Eq. 15, represents a generalization of the three path equations given above. This equation will be reduced to the equation of geodesic (null geodesic upon reparameterization) in the Riemannian geometry, when the parameter $b=0$. It has been shown that Eq. 15 can be used to express trajectories of spinning test particles, massive or massless, in a gravitational field.

\section{THEOREMS ON NULL PATHS IN PAP SPACES}

Let $\Gamma$ and $\Gamma^{\prime}$ be two neighboring null paths of the type given by Eq. 15 defined in PAP space of dimensions $n$. Let $C$ be a point on $\Gamma$ and $C^{\prime}$ be a neighboring point on $\Gamma^{\prime}$. Let $\zeta^{\mu}$ be the vector $C C^{\prime}$ connecting the two points. Let us define the following scalar,

$$
J \stackrel{\text { def }}{=} Z^{\mu} \zeta_{\mu}
$$

where $Z^{\mu}$ is the null tangent to the path (15) defined at $C$. Then we can prove the following theorem:

\subsection{Theorem (I)}

"The scalar $J$ is independent of the position of the point $C$ on the null path $\Gamma$ and is also independent of the choice of the direction $C C^{\prime}$ but depends on the two null paths themselves."

Now, as mentioned in the previous section, the equation of the null paths in the PAP Geometry is given by Eq. 15 and the equation of null geodesic in the Riemannian geometry is given by Eq. 8. It is clear that Eq. 15 tends to Eq. 8, if $b=0$. Keeping in mind the fact that for every absolute parallelism space there exists an associated Riemannian one, we can relate the objects in Eq. 15 to the objects in Eq. 8 by the following relations

$$
\begin{gathered}
Z^{\mu}=\eta^{\mu}(1+g(b)), \\
\tau=p(1+f(b)), \text { and } \\
\zeta_{\mu}=\zeta_{\mu}(1+l(b)) .
\end{gathered}
$$

Here, $g(b), f(b)$ and $l(b)$ are positive functions of the parameter $b$ such that these functions tend to zero when $b$ goes to zero. Now let us evaluate the scalar $J \stackrel{\text { def }}{=} Z^{\mu} \zeta_{\mu}$. Using Eq. 19, we get

$$
\frac{d p}{d \tau}=\frac{1}{(1+f(b))}
$$

For simplicity, we let $G$ for $g(b), F$ for $f(b)$ and $L$ for $l(b)$, which leads to

$$
\begin{aligned}
\frac{d}{d \tau}\left(Z^{\mu} \zeta_{\mu}\right)= & \frac{1}{(1+F)} \frac{d}{d p}\left(Z^{\mu} \zeta_{\mu}\right) \\
= & \frac{1}{(1+F)} \frac{d}{d p}\left[\eta^{\mu}(1+G)\right. \\
& \left.\zeta_{\mu}(1+L)\right] \\
= & \frac{1}{(1+F)}(1+G)(1+L) \\
& \frac{d}{d p}\left(\eta^{\mu} \zeta_{\mu}\right) .
\end{aligned}
$$

Now recalling Eq. 6 and Theorem (I) of KMW, we get

$$
\begin{aligned}
\frac{d}{d \tau}\left(Z^{\mu} \zeta_{\mu}\right) & =0 \\
\text { i.e., } \quad Z^{\mu} \zeta_{\mu} & =\text { constant. }
\end{aligned}
$$

This result proves Theorem (I) on the null path of the PAP space. Now, as an extension of the idea of null path $\Gamma$ passing through a given point $C$ in the PAP space $T_{n}$, one can find $\infty^{n-1}$ of null paths, in the neighborhood of the point $C$, parallel to the null path $\Gamma$. The second theorem can be stated as follows:

\subsection{Theorem (II)}

For a definite value of the scalar $J$, we have $\infty^{n-2}$ of parallel null paths lying in a subspace $T_{(n-1)}$, which 
intersects a local flat subspace $E_{(n-1)}$ of $(n-1)$ dimensions at the point $C$ in a local (n-2)-dimensions flat subspace $E_{(n-2)}$. This $E_{(n-2)}$ is perpendicular to the projection of the null path $\Gamma$ in $E_{(n-1)}$.

To prove this theorem, we shall assume that there is no singularity at the point $C$ or at any point in its neighborhood. If we assume any rectangular axes at $C$, such that the direction ratios along the null path $\Gamma$ are $\left(\lambda_{1}, \lambda_{2}, \ldots, \lambda_{n}\right)$, then:

$$
\lambda_{1}^{2}+{\lambda_{2}}^{2}+\ldots+\lambda_{n}^{2}=0 .
$$

Therefore the tangent null-vector $Z^{\mu}$ to the null path at $C$ has the components $\left(m \lambda_{1}, m \lambda_{2}, \ldots, m \lambda_{n}\right)$, where $m$ is some constant. If the coordinates of the point $C$ are $\left(x^{1}, x^{2}, \ldots, x^{n}\right)$, and if we take the vector $Z^{\mu}$, which is defined above, as $\left(x^{1}, x^{2}, \ldots, x^{n}\right)$, then the scalar $J$ takes the form:

$$
J=\left(x^{1} \lambda_{1}+x^{2} \lambda_{2}+\ldots+x^{n} \lambda_{n}\right) m,
$$

i.e.,

$$
\left(x^{1} \lambda_{1}+x^{2} \lambda_{2}+\ldots+x^{n} \lambda_{n}\right)=\frac{J}{m} .
$$

Due to Theorem (I), $J$ remains constant wherever $\left(x^{1}, x^{2}, \ldots, x^{n}\right)$ lies in the hyperplane. That is, for any other point $C^{\prime}$ on the null path $\Gamma^{\prime}$, parallel to $\Gamma$, of coordinates $\left(x^{1}+k \lambda_{1}, x^{2}+k \lambda_{2}, \ldots, x^{n}+k \lambda_{n}\right)$, where $k$ is a variable parameter depending on the null path $\Gamma^{\prime}, J$ remains constant. Therefore, if we take the components of $Z^{\mu}$ at the point $C^{\prime}$ on the null path $\Gamma^{\prime}$ to be $\left(x_{1}+k \lambda_{1}, x_{2}+k \lambda_{2}, \ldots, x_{n}+k \lambda_{n}\right)$, and substitute in Eq. 25, we have

$$
J=m\left(x^{1} \lambda_{1}+k{\lambda_{1}}^{2}+x^{2} \lambda_{2}+k{\lambda_{2}}^{2}+\ldots+x^{n} \lambda_{n}+k \lambda_{n}{ }^{2}\right) .
$$

Using Eq. 24 we get the same Eq. 25. This means that any one of the set of the null paths parallel to the null path $\Gamma$ must lie in the hyperplane given by Eq. 26, in order to keep $J$ constant. Now if we put $x^{1}=0$, it follows directly that all the points in which these null paths cut any local subspace $E_{(n-1)}$ lie in a local subspace $E_{(n-2)}$ given by

$$
\left(x^{2} \lambda_{2}+\ldots \ldots+x^{n} \lambda_{n}\right)=\frac{J}{m}
$$

This $E_{(n-2)}$ is perpendicular to the null path $\Gamma$ whose projection in $E_{(n-1)}$ is given by

$$
\frac{x^{2}}{\lambda_{2}}=\ldots=\frac{x^{n}}{\lambda_{n}} .
$$

Hence, the second theorem is proved.

Using the two previous theorems and the same idea of KMW, we can write a general expression of the redshift. This expression depends essentially on the idea of

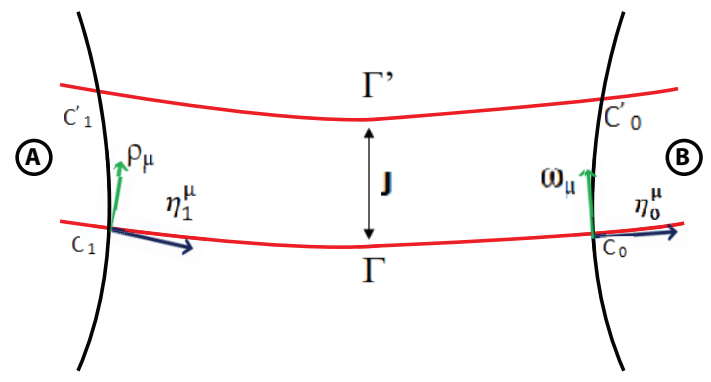

Fig. 1. - Paths and Null-Pathes of the emitter and the reciever.

the wave fronts, which are represented by a set of parallel null paths passing through the points of the wave front. Their projections in the local subspace are perpendicular to this wave front (see Fig. 1). Therefore, the actual wavelength is determined by the perpendicular distance between the wave fronts corresponding to two parallel sets of null paths. In other words, it is the interval between the points of intersection of the two local subspaces defined by the two sets of parallel null paths and the observer's world line.

\section{GENERAL EXPRESSION OF REDSHIFT IN PAP SPACE}

In the previous section, we have shown that the two theorems on null geodesics proved by KMW are also applicable to the null paths given by Eq. 15. Now, we are going to assume that the trajectory of a photon with spin one in a gravitational field is spin-dependent and given by Eq. 15. So, we can easily establish a general formula for the redshift of spectral lines similar to that given by KMW.

Consider a null path of the form of Eq. 15 connecting the two points $C_{1}$ and $C_{0}$ at which the emitter $A$ and receiver $B$ are located, respectively. The null path $\Gamma$ belongs to the same wave front observed by $A$ and $B$, as shown in Fig. 1. Let $\eta_{1}{ }^{\mu}$ and $\eta_{0}{ }^{\mu}$ be the components of the transport null tangent to $\Gamma$ at $C_{1}$ and $C_{0}$, respectively. Let $\Gamma^{\prime}$ be a null path parallel to $\Gamma$, belonging to the succeeding wave front, intersecting the world lines of $A$ and $B$ at $C_{1}^{\prime}$ and $C_{0}^{\prime}$, respectively. If $\lambda_{1}$ and $\lambda_{0}$ are the wavelengths of the same spectral line as it is given by the emitter $A$ and received by the reciever $B$, respectively, then the components of the vectors $C_{1} C_{1}{ }^{\prime}$ and $C_{0} C_{0}{ }^{\prime}$ are $\rho^{\mu}, \varpi^{\mu}$, which represent the components of the unit tangents to the world lines of $A$ and $B$, respectively. These unit tangents are solutions of Eq. 15 for $A$ and $B$, upon taking $b=0$. The vectors $\rho^{\mu}$ and $\varpi^{\mu}$ are the values of the vector $\xi^{\mu}$ in Theorem (I) evaluated at $C_{1}$ and $C_{0}$, respectively, while $\eta_{1}{ }^{\mu}$ and $\eta_{0}{ }^{\mu}$ represent the values of the vector $Z^{\mu}$, of the same theorem, evaluated at $C_{1}$ and $C_{0}$ as stated above. Now applying Theorem (I) and equating the values of $J$ at $C_{1}$ and at $C_{0}$ we get 


$$
\begin{aligned}
\lambda_{1} \rho_{\mu} \eta^{\mu}{ }_{1} & =\lambda_{0} \varpi_{\mu} \eta^{\mu}{ }_{0} \\
\text { i.e., } \frac{\lambda_{0}}{\lambda_{1}} & =\frac{\rho_{\mu} \eta_{1}^{\mu}}{\varpi_{\mu} \eta^{\mu}{ }_{0}} .
\end{aligned}
$$

Thus,

$$
\frac{\Delta \lambda}{\lambda_{1}}=\frac{\lambda_{0}-\lambda_{1}}{\lambda_{1}}=\frac{\rho_{\mu} \eta_{1}^{\mu}}{\varpi_{\mu} \eta_{0}^{\mu}}-1
$$

This gives a general formula for the redshift of spectral lines coming from a distant object.

\section{CONCLUDING REMARKS}

In the present work, we have investigated the validity of two important theorems on null geodesics in the context of the PAP geometry. These two theorems are important to establish a general formula for the redshift of spectral lines especially when the trajectories of massless particles are spin dependent. Eq. 15 is the equation representing such trajectories. This equation can be used as an equation of motion in the context of any field theory written in the AP-geometry including GR (Wanas 1990).

In conclusion we can write the following general remarks:

(1) In the present work, we tried, as far as we could, to use the same notations, as those used in the original work of KMW in order to facilitate comparison.

(2) The path Eq. 15 can be used to represent the trajectory of a test particle or the trajectory of a massless particle, in a background gravitational field, upon adjusting the parameter $\tau$. The right hand side of this equation is suggested to represent a type of interaction between the spin of the moving particle and the torsion of the background gravitational field. The parameter (b) is a spin dependent parameter (Wanas 1998).

(3) The two theorems on (15), when it represents null paths, can be reduced to the original KMW theorems, reviewed in Section 2, upon taking $b=0$.

(4) Eq. 30 gives the redshift taking into account the spin-torsion interaction. This equation appears to be the same as that given by KMW, but the main difference is that the effect of the spin of the moving particle will appear in the values of the null vectors $\eta_{0}{ }^{\mu}$ and $\eta_{1}{ }^{\mu}$ which are solutions of Eq. 15 and not of the equation of a null geodesic.

(5) KMW used formula (7) to get the Doppler shift. In an attempt to interpret the solar limb effect (Mikhail, et al. 2001), the use of Eq. 7 has been widened to account, not only for the relative radial velocity between $A$ and $B$ (Doppler-shift), but also for:

(i) The effect of gravity (gravitational redshift).

(ii) The effect of the direction of the null-geodesic.

In addition to these effects, the formula (30) will account also for the effect of the spin-torsion interaction on the value of the redshift.

(6) Formula (30) can be used to get the redshift whether it is treated as a metric phenomena or not. In future works, the redshift of spinning particles coming from some celestial objects as pulsars and binary pulsar, will be calculated.

\section{ACKNOWLEDGMENTS}

The corresponding author would like to thank the JKAS team including all scientific reviewers, editorial board and staffs. I would like to thank Prof. HeeWon Lee (the scientific editor of this paper) for his kind help in correcting some typo errors by himself. I cannot forget to thank Ms. Yeongiu Jung, for her useful and accurate comments which made the manuscript improved.

\section{REFERENCES}

Adler, R., Bazin, M., \& Schiffer, M. 1975, Introduction to General RelativityDe Rujula, A. 1987, 2nd edn. (New York: McGraw Hill)

Bażański, S. L. 1977, Dynamics of Relative Motion of Test Particles in General Relativity, Ann. Inst. H. Poincaré, A27, 145

Bażański, S. L. 1989, Hamilton-Jacobi Formalism for Geodesics and Geodesic Deviations, J. Math. Phys., 30, 1018

Colella, R., Overhauser, A. W., \& Werner, S. A. 1975, Observation of Gravitationally Induced Quantum Interference, Phys. Rev. lett., 34, 1472

De Rujula, A. 1987, May a Supernova Bang Twice?, Phys. Lett. B, 193, 4, 514

Kermak, W. O., McCrea, W. H., \& Whittaker, E. T. 1933, On Properties of Null Geodesics and the Application to the Theory of Radiation, Proc. Roy. Soc. Edin., 53, 31

Mikhail, F. I., Wanas, M. I., \& Morcos, A. B. 2001, Application of Theorems on Null-Geodesics on The Solar Limb Effect, Astrophys. Space Sci., 280, 223, gr-qc/050513

Overhauser, A. W., \& Colella, R. 1974, Experimental Test of Gravitationally Induced Quantum Interference, Phys. Rev. lett., 33, 1237

Schramm, D. N., \& Truran, J. W. 1990, New Physics from Supernova 1987A, Physics Report, 189, 89

Wanas, M. I. 1990, Notes on Applications of General Relativity in Free Space, Astron. Nachr., 311, 253

Wanas, M. I., Melek, M., \& Kahil, M. E. 1995, New Path Equations in Absolute Parallelism Geometry, Astrophys. Space Sci., 228, 273, gr-qc/0207113

Wanas, M. I. 1998, Motion of Spinning Particles in Gravitational Fields, Astrophys. Space Sci., 258, 237, gr-qc/9904019

Wanas, M. I. 2000, Parameterized Absolute Parallelism: A Geometry for Physical Applications, Turk. J. Phys., 24, 473, gr-qc/0010099 
Wanas, M. I., Melek, M., \& Kahil, M. E. 2000, Quantum Interference of Thermal Neutrons and SpinTorsion Interaction, Grav. Cosmology, 6, 319, grqc/9812086

Wanas, M. I. 2002, Quantum Consequences of Parameterizing Geometry, Proc. MG IX., Part B, 1303

Wanas, M. I., Melek, M., \& Kahil, M. E. 2002, SN1987A: Temporal Model, Proc. MG IX., Part B, 1100, gr-qc/0404115

Weber, J. 1994, Supernova 1987A Rome Maryland Gravitational Radiation Antenna Observations., Proc. First Edoardo Amaldi Conf. on "Gravitational Wave Experiment", Ed. E. Coccia et al., World Scientific, 416

Werner, S. A., Kaiser, H., Arief, M., \& Clothier, R. 1988, Neutron Interference Induced by Gravity: New Results and Interpretations, Physica, B151, 22 